\title{
AIR-TO-GROUND LASERCOM SYSTEM DEMONSTRATION
}

\author{
Frederick G. Walther, George A. Nowak, Steven Michael, Ronald Parenti, Jeffrey Roth., John Taylor, \\ William Wilcox, Robert Murphy, Joseph Greco, John Peters, Timothy Williams, Scott Henion, \\ Richard Magliocco, Tom Miller, and Alicia Volpicelli
}

Massachusetts Institute of Technology Lincoln Laboratory

Lexington. MA

\begin{abstract}
This article presents an overview of the air to ground lasercom demonstration performed under the Free-Space Optical Communications Airborne Link (FOCAL) program. Techniques used to mitigate fading, demonstrated in 2008 and reported earlier, are reviewed as are the basic equipment approach, also reported earlier. We overview the new results for tracking, fiber coupling, channel measurements and communications performance for the air-to-ground link.
\end{abstract}

This work was sponsored by the Department of Defense, RRCO DDR\&E, under Air Force Contract FA8721-05-C-0002. Opinions, interpretations, conclusions and recommendations are those of the authors and are not necessarily endorsed by the United States Government.

\section{INTRODUCTION}

An important future application of lasercom is support of tactical ISR missions, such as persistent surveillance, delivering near real-time sensor data to the operational theater. Representative objective requirements include the ability to transport data at rates up to tens of $\mathrm{Gb} / \mathrm{s}$ over a range of roughly $50 \mathrm{~km}$. The air terminal should have low $\mathrm{SWaP}$ and low integration impact on the aircraft. Use of standard terrestrial network protocols can simplify the implementation, particularly at the client interface. A key advantage of lasercom is its ability to provide high bandwidth without adding to rf congestion in theater.

\section{BASIC ELEMENTS OF A LASERCOM SYSTEM}

Optical communications based on single-mode fibers in the 1550 $\mathrm{nm}$ band is commonly used in commercial telecom and provides a readily available base of components including efficient preamplified receivers. For this reason, $1550 \mathrm{~nm}$ has become the wavelength of choice for free-space communication systems that utilize single-mode detection.

In free-space architectures the fiber connectivity between the transmitter $(\mathrm{Tx})$ and receiver $(\mathrm{Rx})$ is replaced with a power delivery subsystem, the sole purpose of which is to deliver power from the transmit fiber to the receive fiber as efficiently as possible. Communications performance is based on both the mean power in fiber as well as the statistics of the fluctuation of power in fiber. Design approaches must maximize average power and minimize its variance in the presence of atmospheric turbulence and platform jitter. A graphical illustration of the essential hardware components in a representative free-space optical data link is shown in Fig. 1.

At optical wavelengths, phase distortions imposed by atmospheric turbulence cause strong fluctuations in the far-field intensity of a laser beam transmitted over a free-space link. Phase distortions within the Rayleigh range of the transmitter and receiver planes can be corrected through the use of highbandwidth tracking and adaptive-optics,[1] but errors accumulated between these two regions cannot be sensed or

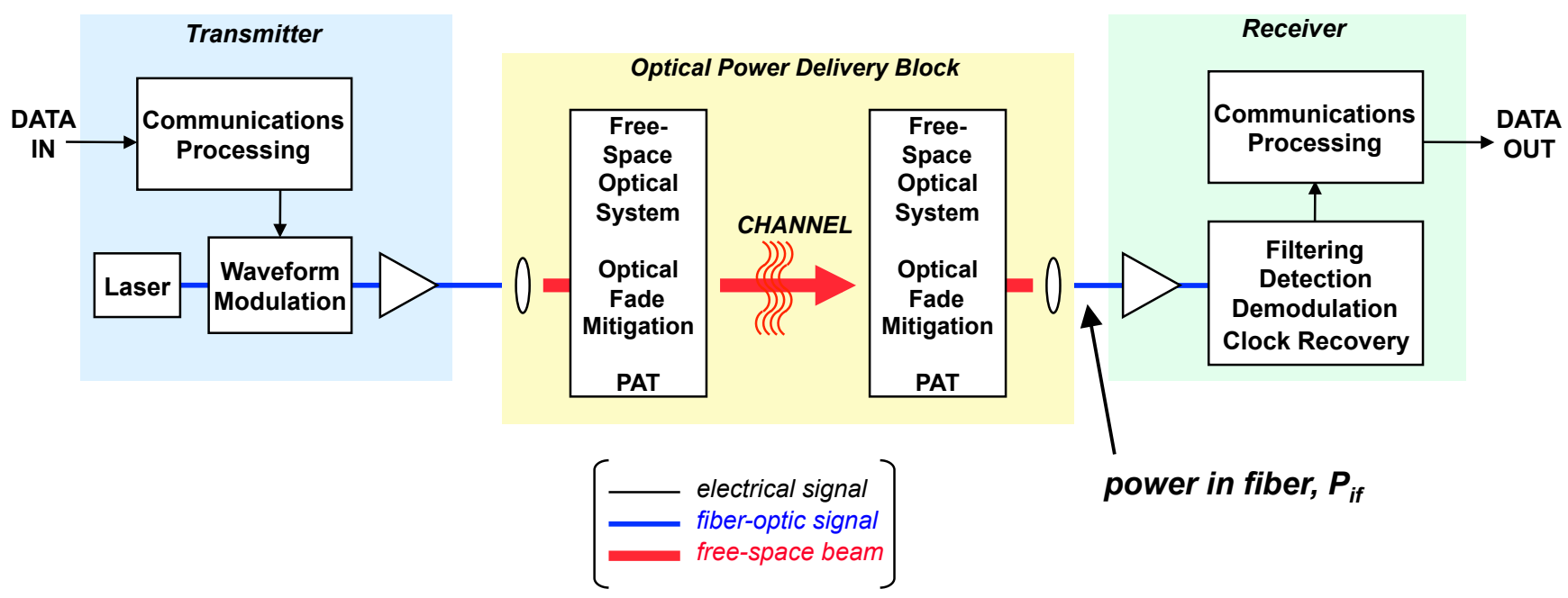

Figure 1. Principal components of a free-space optical data link. A high-bandwidth pointing, acquisition, and tracking (PAT) system is essential for maintaining good beam alignment in both the transmitter and the receiver. 
corrected. The effects of uncompensated signal fading due to beam scintillation and fiber coupling losses must be dealt with through alternative means; examples of ancillary mitigation techniques that can be applied at the physical layer include timedelayed diversity (TDD),[2] forward error correction (FEC),[3] and data interleaving.[4]

In recent years, several experimental teams have demonstrated the feasibility of transmitting gigabit-class data rates over longpath free-space links. A notable example is the DARPA-funded ORCA program, which is evaluating the performance of transceivers that incorporate adaptive-optics compensation at both the transmitter and receiver nodes. [5,6] The dimensions of the apertures used in those tests were of the order of $10 \mathrm{~cm}$.

The optical mitigation approach that Lincoln Laboratory has pursued incorporates a single transmitter and multiple smallaperture receivers, each of which is individually tilt-corrected using a high-bandwidth tracking device. The separation distance between the apertures is large enough to ensure near-total decorrelation under most turbulence conditions, and since all of the apertures are small compared to the phase coherence diameter $\left(\mathrm{r}_{\mathrm{o}}\right)$ a high coupling efficiency into a single-mode fiber can be consistently maintained. The incoherent sum of the electrical signals produces a composite data stream, which exhibits a variance that is inversely proportional to the number of uncorrelated detector outputs.

Under typical turbulence conditions the projected beam at $50 \mathrm{~km}$ is nearly diffraction-limited and has a diameter of approximately $8 \mathrm{~m}$. A beam profile of this size permits the implementation of multiple wide field-of-view transceivers at the aircraft to avoid outages due to structural blockage, and also enables system architectures that switch between conformal apertures having limited fields-of-regard. Since spatial averaging is not significant for small apertures, beam scintillation as measured at a point is the dominant effect of atmospheric turbulence. As discussed in the next section, the FOCAL system incorporates a combination of real-time FEC and interleaving techniques to minimize the effects of residual signal fading.

\section{SCINTILLATION MITIGATION APPROACHES}

The first scintillation mitigation technique used in this demonstration is optical spatial diversity, which is shown here for the receiver. This technique was demonstrated in a $5.4 \mathrm{~km}$ horizontal link experiment in September 2008 and reported previously.[7] For the FOCAL air-to-ground experiment described here, the aircraft aperture was increased to $2.5 \mathrm{~cm}$ to accommodate longer ranges; however, the four $1 \mathrm{~cm}$ ground apertures were not changed. Using fiber add-drop multiplexers, separate beacon wavelengths were multiplexed into the ground receive apertures to serve as uplink tracking beacons. The downlink signal was separately detected in each aperture chain, with signals then summed for clock recovery and bit detection. The receiver filter bandwidth was $10 \mathrm{GHz}$. A dynamic variable optical attenuator (VOA) was included in each detection circuit to reduce signal dynamic range at the decision circuits.

Optical diversity ideally reduces the log fade level by the number of apertures if beams are statistically independent; this experiment employed four receiver apertures. Achieving that independence requires separating the beams by a distance greater than the intensity coherence scale size. In our previous work, we showed that $10 \mathrm{~cm}$ aperture separations were sufficient for turbulence considered representative of flight conditions. The rhomboidal aperture pattern shown below permitted an accurate evaluation of intensity coherence length; the receiver separations

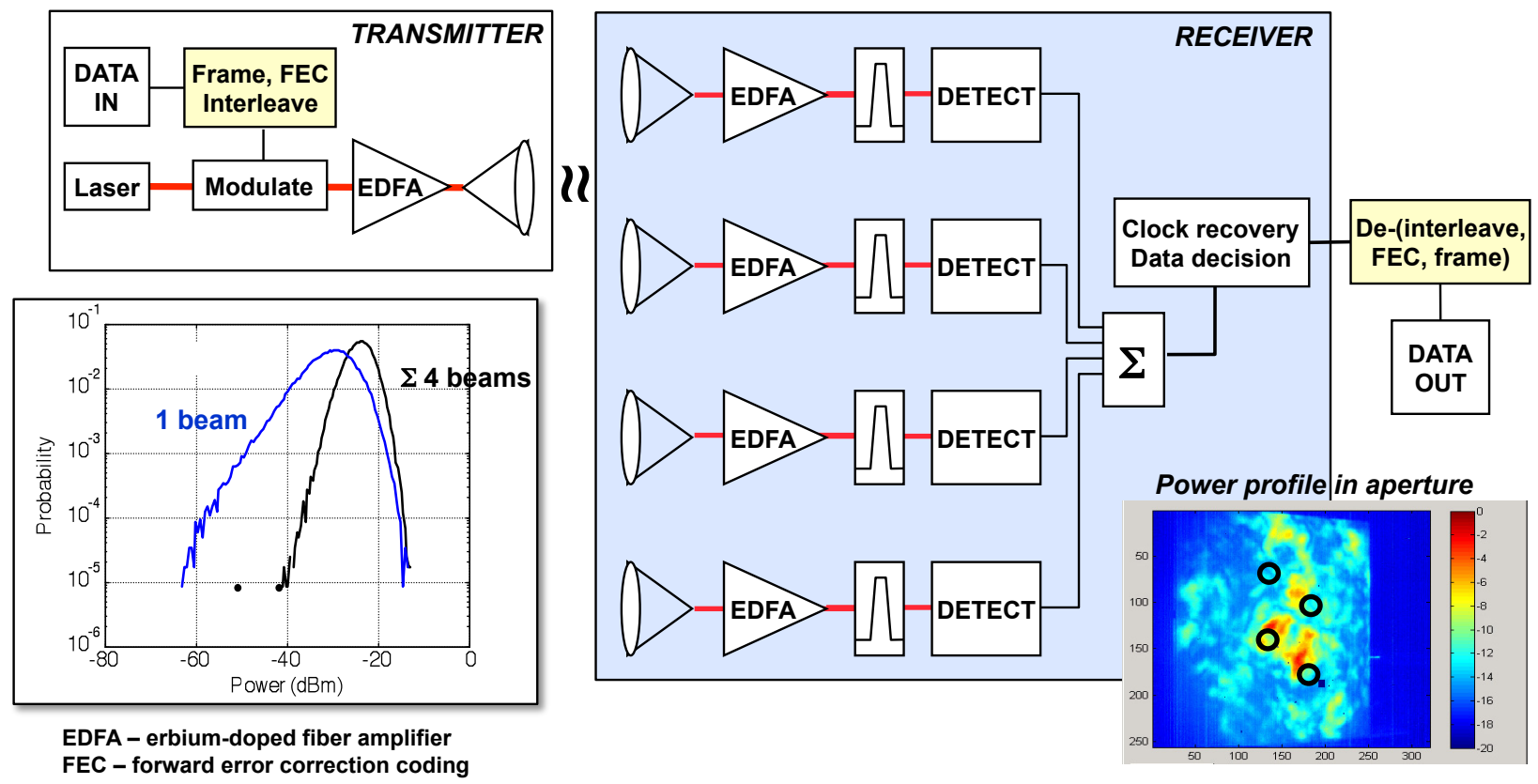

Figure 2. The downlink path for the FOCAL experiment employed a single transmitter mounted in the aircraft and four independently-tracked detectors in the ground-based receiver. The receiver outputs were incoherently summed, and the resulting improvement is demonstrated by the distributions shown in chart on the lower left-hand side of this figure. The detector layout and an image of the scintillation pattern is shown in the picture on the lower right-hand side of this figure. 
ranged from 10 to $20 \mathrm{~cm}$. A system block diagram and examples of collected data are shown in Fig. 2. Note that spatial diversity applies to both up and down links.

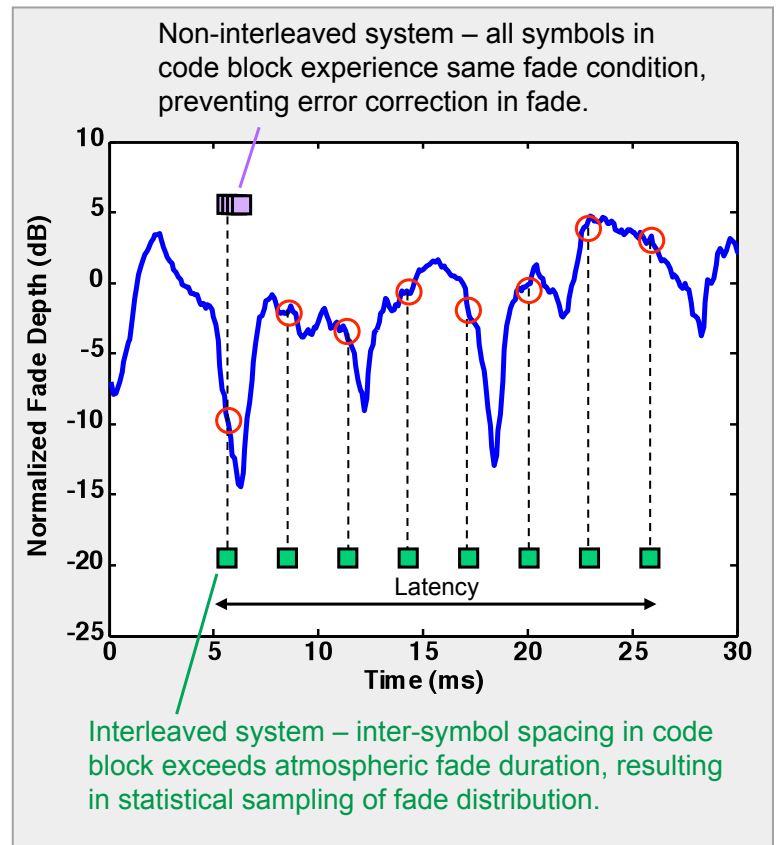

Figure 3. Interleaving refers to a technique in which a code block is segmented into a string of symbols that are temporally separated in order to minimize the likelihood that an entire block will be transmitted during a channel fade. The use of encoding allows the entire code block to be recovered, even if some of the symbols are decoded incorrectly.

The second scintillation mitigation technique used on the downlink is forward error correction with interleaving; the basic interleaving concept is illustrated in Fig. 3. FEC adds symbols to each codeword, thereby allowing the recovery of all symbols if some are lost due to fading. The Reed-Solomon $(255,239)$ code used can correct 8 byte (symbol) errors per codeword. For OTU1 framing with RS(255,239) FEC, 64 codewords of 255

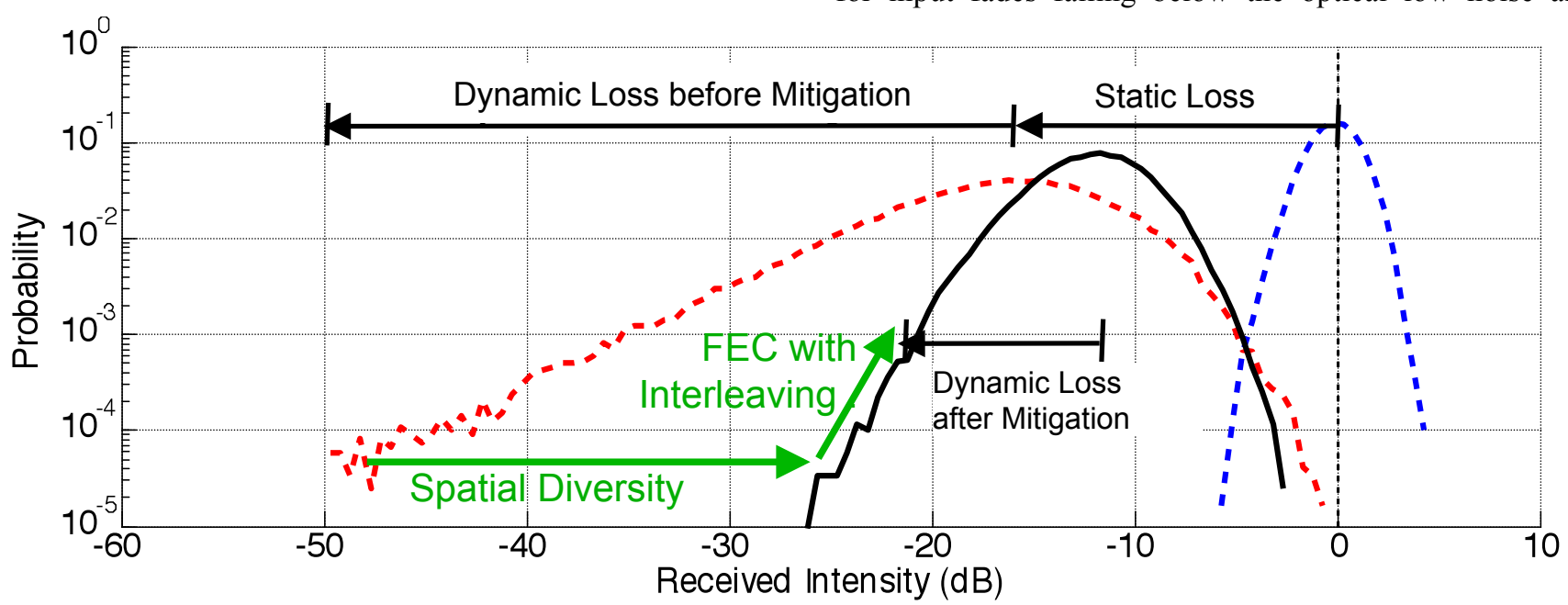

Figure 4. Comparison of received signal distribution functions for a short link with low turbulence (blue curve), a unmitigated long link with high turbulence (red curve), and the long link with scintillation mitigation (black curve). Optimized mitigation schemes can reduce the transmitted signal power requirement by more than $20 \mathrm{~dB}$. bytes each constitute a $\sim 50 \mu$ s frame. This is far shorter than a millisecond class atmospheric fade. If all symbols of the codeword fall in the fade, the FEC is ineffective.

Interleaving provides temporal diversity by spacing the code symbols by a time duration in excess of the characteristic atmospheric fade.[8] For this work, the symbols of each codeword were spaced by $5 \mathrm{~ms}$, leading to a latency of 1.25 seconds after deinterleaving.[9]

The combination of an optical mitigation scheme to reduce scintillation-induced fading and an FEC code operating with sufficient interleaving in the data domain can make communications in the deep fading channel tractable, which significantly extends the available range for air-to-ground communications. Fig. 4 illustrates the improvement achieved in the mean power level (referred to as the static loss) and the reduction of the signal fluctuations (referred to as the dynamic loss). Under severe turbulence conditions, the combined effect of spatial diversity, interleaving, and encoding can easily reduce the transmitted signal requirement by more than $20 \mathrm{~dB}$.

\section{DESCRIPTION OF THE TERMINAL HARDWARE}

Fig. 5 illustrates the optical layouts used for the transmit and receive terminal implementations of the electro-optic and optical modules. A single optical fiber is used in both transmit and receive EO module designs, with transmit/receive diplexing done in the fiber domain. This approach reduces complexity of optical hardware and is enabled by the negligible point-ahead angle in air-to-ground links. In the transmitter (aircraft) E-O module, the transmit/receive diplexer is implemented with a circulator where higher powers would be expected.

The receiver diagram illustrates one channel of a four-channel ground receiver. Here a simple fiber add/drop multiplexer (ADM) is used as the beacon/receiver diplexer to provide isolation against beacon back-reflection from the fiber collimator. A Bragg grating provides $10 \mathrm{GHz}$ passband filtering following the preamplifier. A dynamic VOA helps stabilize the signal delivered to the clock recovery and bit decision circuits for input fades falling below the optical low noise amplifier 

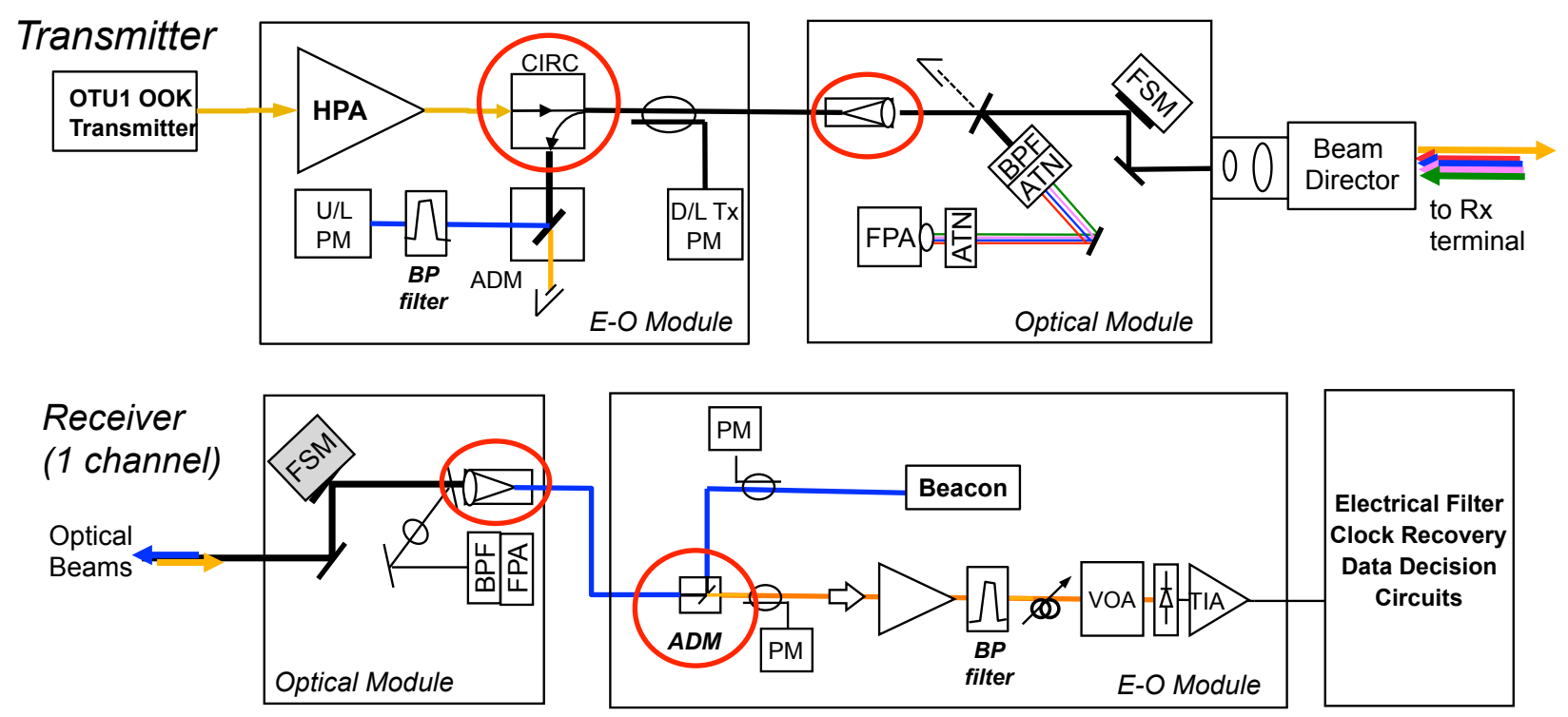

Figure 5. Subsystem elements of the FOCAL transceiver showing the transmit/receive diplexing elements and add-drop multiplexer. To reduce cost and complexity, common-module construction was applied in the transmitter and receiver subsystem designs.
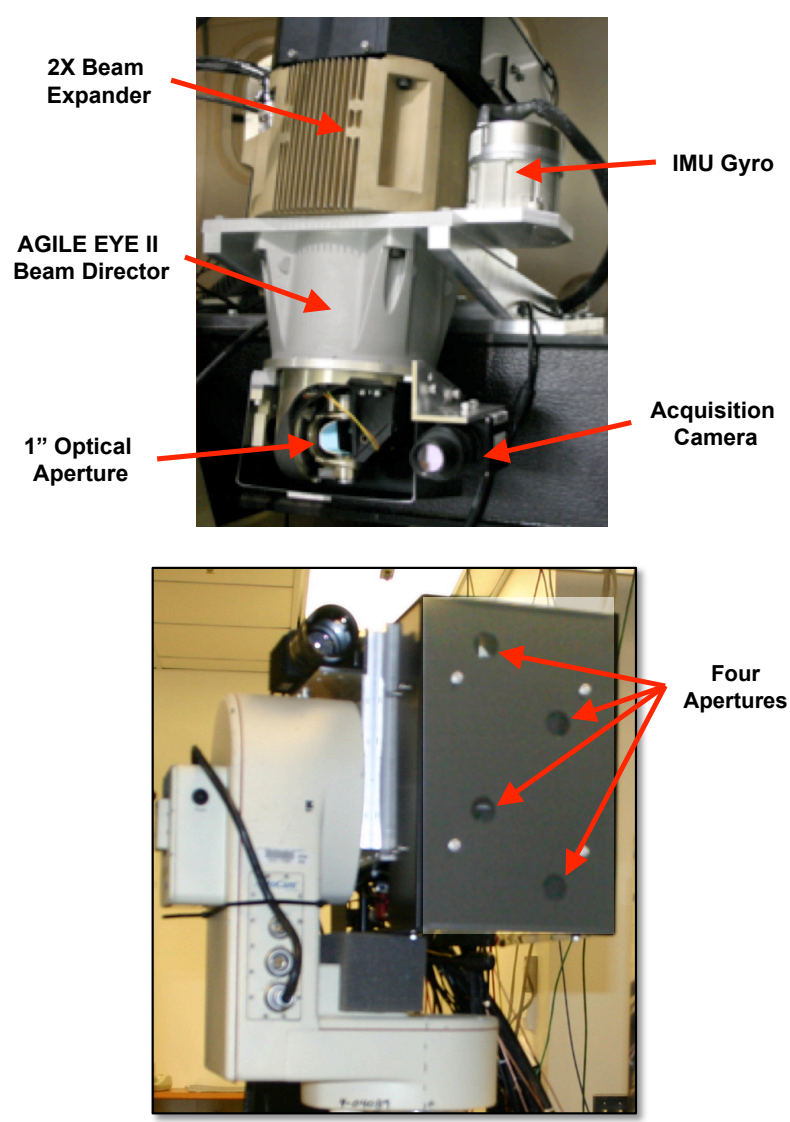

Figure 6. Picture of the aircraft-mounted optical module (upper figure) and ground-based optical module (lower figure). The four ground-based receivers are independently pointed by separate tracking units.

(LNA) saturation level. In the four-channel receiver the transimpedance amplifier (TIA) outputs are summed before delivery to the decision and clock recovery circuits. The optical modules for both ends of the link are essentially common in design, except for filter pass-band wavelengths.

Beam-control hardware used in the airborne and ground terminals is illustrated in Fig. 6. In the aircraft, the common optical fiber couples through an optical collimator. A fast steering mirror (FSM) directs the beam through the floor of the module to a beam expander. An electrically adjustable splitter directs a portion of the incoming uplink tracking beams to a tracking camera, the processed output of which drives the FSM to maintain a constant uplink centroid on the track camera. A flip-in retro-reflector allows boresighting of the fiber to the tracking camera by adjusting the beam splitter in tip/tilt. A flipin beam spoiler broadens the downlink beam during acquisition.

The optical module integrates to a commercial (BAE AEII) beam director that provides a $2.5 \mathrm{~cm}$ output beam steerable over a hemisphere; however, the unit is mounted inside the aircraft with a view through an optical window so that its field-of-regard is restricted. An IMU/gyro, part of an Applanix system, is rigidly mounted to the beam director to allow stable pointing in the presence of aircraft motion. A wide field-of-view acquisition camera is also mounted to the unit with fixed view through the window, both to aid in acquisition as necessary and to document the aircraft's view of the ground.

A similar optical module is used in the ground terminal, which is also depicted in Fig. 6. The beam path following the FSM is directly in object space. No further optics beyond the fiber collimator are used on the ground. One stabilization technique, reported previously, [10] is to dither the FSM in a conical scan. This allows the incoming fiber power to be detected synchronously, thus providing a nutation tracking discriminant for boresight alignment of the fiber to the camera by adjusting the beam splitter during operation. As with the aircraft optical module, a flip-in retro-reflector provides the means of initially boresighting the fiber to the tracking camera.

Two such modules are assembled on each side of a plate, and 

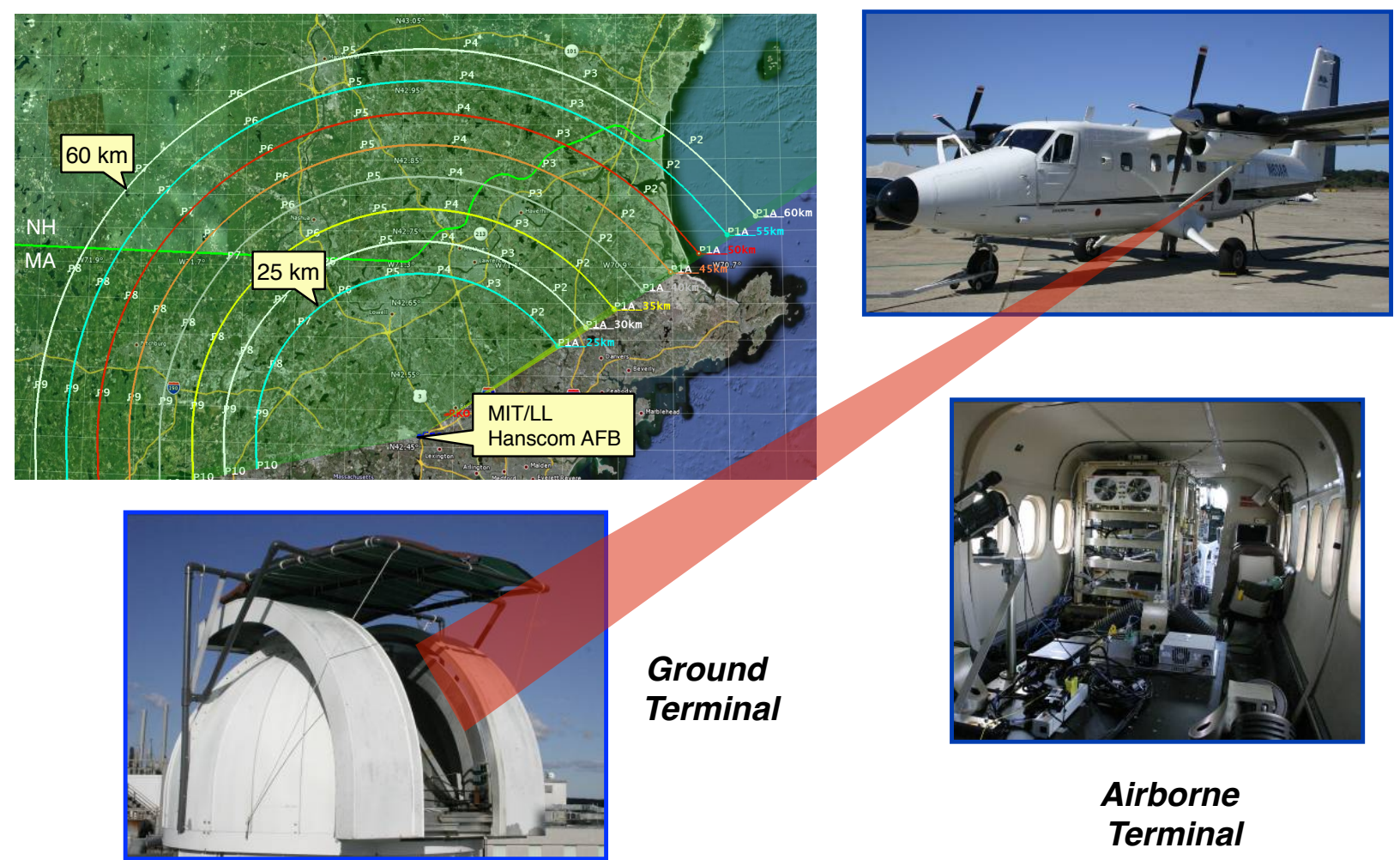

Ground

Terminal

\section{Airborne Terminal}

Figure 7. In the FOCAL experiment the performance of a $2.7 \mathrm{~Gb} / \mathrm{s}$ link between a transmitter mounted in a Twin Otter aircraft and a ground-based receiver was evaluated. For most of the tests, the aircraft was flown in a semi-circular pattern centered on the location of the ground terminal. Tests were performed at ranges between 25 and $60 \mathrm{~km}$.

two such plates are combined to form the 4-aperture ground module. The unit is integrated with a commercial pan-tilt unit to form a fully steerable opto-mechanical system. A broad uplink beacon and a wide field-of-view acquisition camera are also integrated in the pan-tilt unit.

\section{FREE-SPACE OPTICAL COMM DEMONSTRATION}

Key aspects of the FOCAL flight demonstration conducted during September and October of 2009 are described in Fig. 7. This work extended the horizontal link demonstrations of 2008 to an air-to-ground flight demonstration.

As described earlier, the source optical module combined with a hemispherical beam director was integrated into a Twin Otter aircraft leased by Lincoln Laboratory. A ground terminal incorporating spatial diversity was integrated into an available optical dome on the roof of a laboratory building, with a ground control room beneath. For the demonstration, the aircraft flew arcs roughly north of Hansom Field at ranges from 20 to $60 \mathrm{~km}$. To characterize operation, we measure tracking performance, fiber coupling efficiency, channel turbulence and communications performance as a function of slant range, elevation angle and time of day.

A good indication of the performance of the beam tracking subsystems is the fiber coupling efficiency, which is examined in the plots shown in Fig. 8. These distribution functions provide a comparison of the received power statistics for the full collection aperture and the single-mode fiber that couples photons to the communications detection device. In the raw data (dashed green curve) the low-signal tail displays an anomalous behavior due to tracking errors caused by a gimbal stiction problem, which has since been corrected. In the processed data (solid green curve), the time intervals that were impacted by the stiction problem have been eliminated from the analysis. A comparison of the full-aperture power statistics (black curve) and the processed power-in-fiber statistic provides a fair

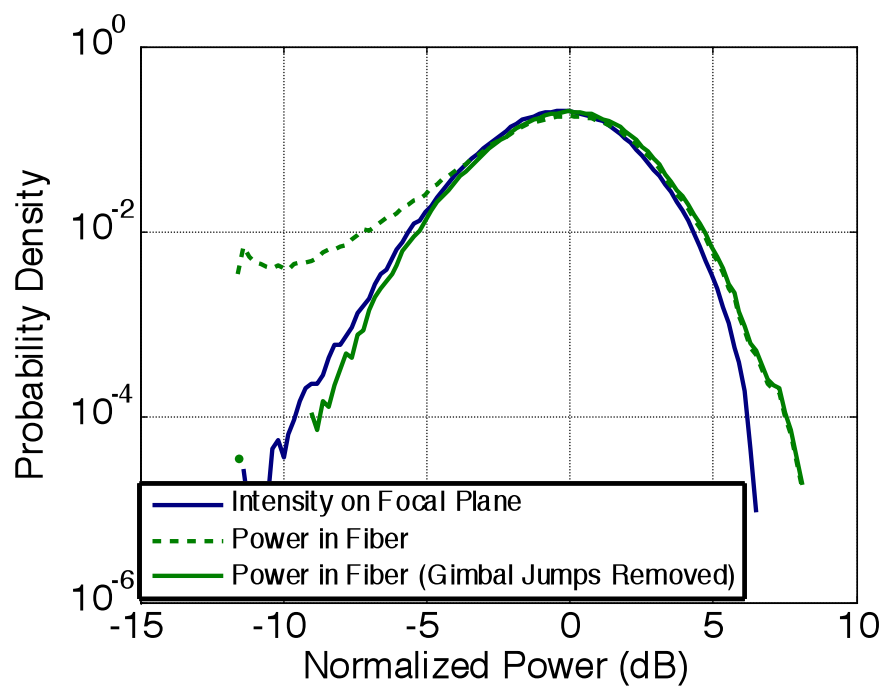

Figure 8. Coupling efficiency can be quantified by comparing the power collected by the full aperture and the single-mode fiber. Excluding intervals during which tracking errors caused by mechanical stiction were anomalously high, the coupling losses were estimated to be $1.5 \mathrm{~dB}$ 
measure of the system's dynamic (turbulence-induced) coupling loss, which is estimated to be $1.5 \mathrm{~dB}$.

The data collected during the FOCAL experiment included a variety of measurements intended to assist in the understanding of the atmospheric channel and the functionality of the transceiver hardware. Fig. 9 illustrates two sample results from the channel scintillations measurements. These distributions illustrate the single and combined 4-aperture statistics for two cases: $50 \mathrm{~km}$ afternoon and $25 \mathrm{~km}$ evening conditions. Overlaid on these charts are wave propagation model simulations for the respective cases, scaled with a multiplier for best fit relative to the Hufnagel-Valley $\mathrm{HV}_{5,7}$ model. Afternoon conditions fit well to a curve derived for $1.1 \mathrm{xHV}$, while evening conditions are best described as having a $0.7 \mathrm{xHV}$ profile. Over the range of conditions measured, from 20 to $60 \mathrm{~km}$ and for various times of day, the HV channel model fits the data with multipliers from 0.4 to 2 .
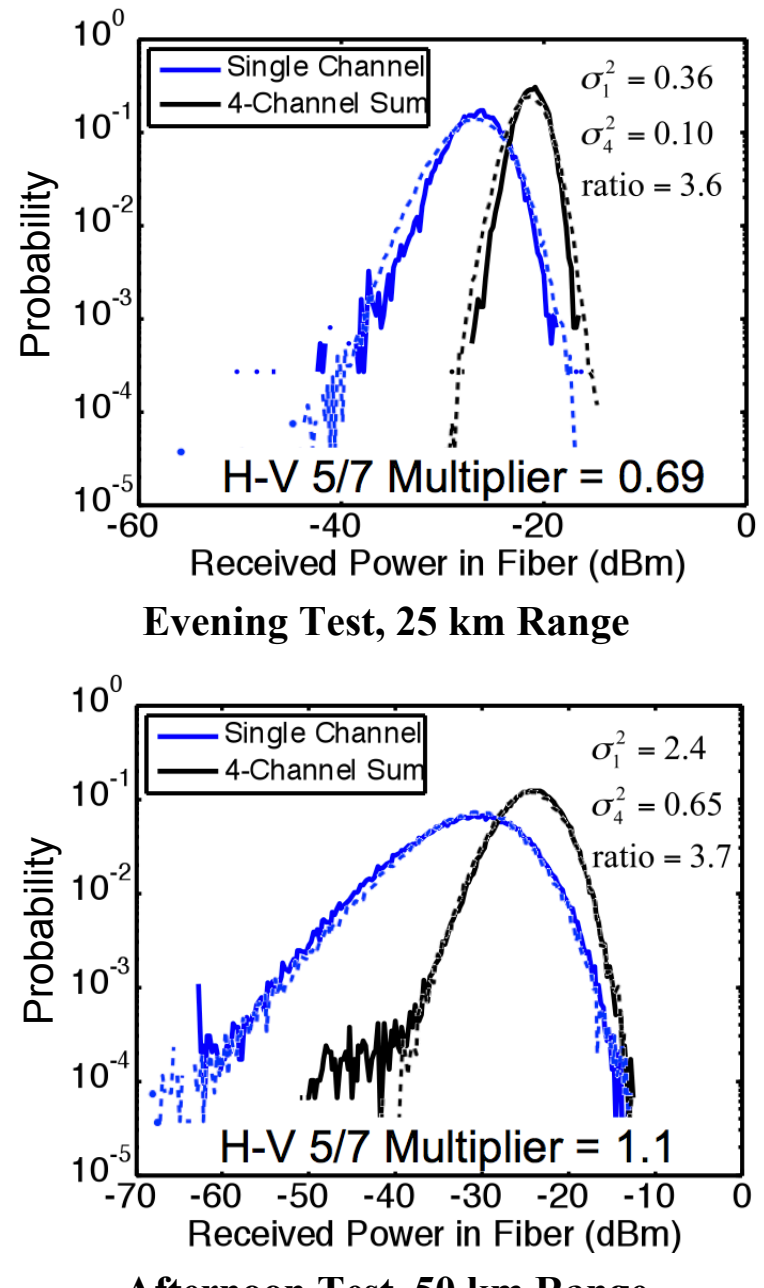

\section{Afternoon Test, 50 km Range}

Figure 9. Comparison of measured power-in-fiber distribution functions with predictions obtained from numerical simulations using the $\mathrm{HV}_{5,7}$ turbulence model. Good agreement was obtained following the application of near-unity multiplicative factors to the turbulence strength profile.

Note that imperfect tracking tends to increase the apparent scintillation fading and leads to overestimates of channel turbulence. Overall, spatial diversity reduces scintillation fading about as expected.

\section{COMMUNICATIONS PERFORMANCE}

One metric of communications performance is the ability to deliver error-free data files of significant length. For this demonstration the basic unit was a six-minute file containing 100 Gbytes of image data. The link delivered multiple transfers of this file with no dropped bits at a range of $25 \mathrm{~km}$ from an altitude of $12 \mathrm{kft}$. The fading strength for this geometry is comparable to that for a $37 \mathrm{~km}$ link to an aircraft flying at $18 \mathrm{kft}$.

The six-minute block was selected to assure reasonable probability of cloud free line of sight (CFLOS) for a daytime link operating in New England. Not all days could provide CFLOS for that time duration. Cloud conditions generally improved in early morning and early evening. However, turbulence conditions are also more benign then, so several flights probed the channel in late morning and early afternoon as cloud conditions permitted.

Although the original intent of the FOCAL experiment was to demonstrate error-free communications performance out to about $30 \mathrm{~km}$, we extended to longer distances on several flights to evaluate the breakdown of the communication link in a unidirectional data throw. Tracking remained robust out to 60 $\mathrm{km}$, which was the longest range tested. Data retransmission was not used during these experiments.

Standard network test gear and our high-speed codec/interleaver hardware afforded an accurate assessment of the codeword error. The failure criterion used for communications was conservative: a single uncorrectable codeword (out of 64 in the frame) was deemed a failure and the entire frame of 16320 bytes was rejected. The charts below summarize the observed frame-error performance under moderate and strong turbulence conditions.

The dropped frame count recorded for ranges beyond $40 \mathrm{~km}$ is plotted as a function of time and propagation range for two representative flights in Fig. 10; a concise summary of the frame error rate as a function of range is displayed in Table I. We note that all of the FOCAL flights were performed at altitudes below $12 \mathrm{kft}$, which corresponds to an elevation angle of $4^{\circ}$ at a slant range of $50 \mathrm{~km}$. Operation at higher altitudes would reduce the impact of turbulence-induced fading, and yield improved performance for the same propagation range. Significant gains in range capability would also be realized with the application of a link retransmission protocol, since the $2.5 \mathrm{~Gb} / \mathrm{s}$ payload of OTU1 signaling provides a $25 \%$ excess capacity beyond the source application requirement of $2 \mathrm{~Gb} / \mathrm{s}$. Based on a conservative assumption of a $20 \%$ retransmission capability, we estimate that error-free performance to at least $50 \mathrm{~km}$ could be achieved with the system as presently configured.

Table I

Frame Error Rate vs. Propagation Range

\begin{tabular}{|c|c|c|}
\hline Time (turbulence) & Range (km) & Frame error rate \\
\hline Evening (moderate) & $40-48$ & $.0001 \%$ \\
\hline " & $48-56$ & $2 \%$ \\
\hline " & $56-59$ & $10 \%$ \\
\hline Mid-day (strong) & $40-43$ & $5 \%$ \\
\hline
\end{tabular}



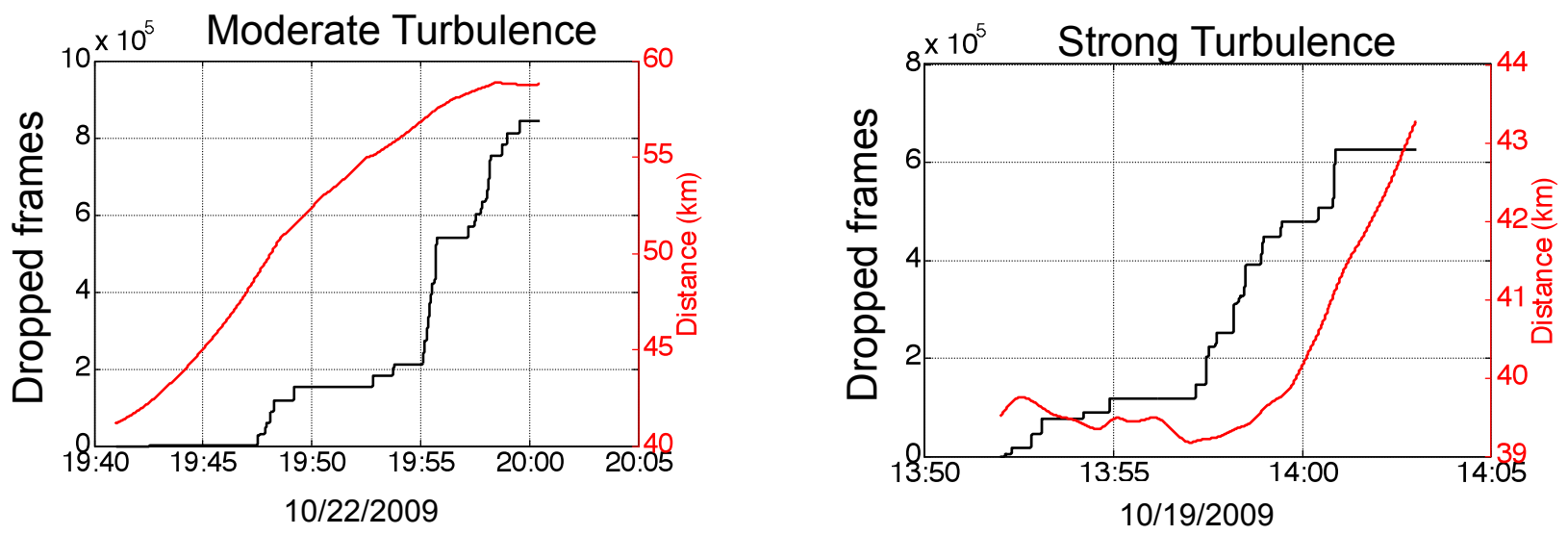

Figure 10. Plot of the dropped frame count and propagation distance as a function of time for two representative FOCAL flights.

\section{SUMMARY OF RESULTS}

The FOCAL demonstration conducted in the fall of 2009 successfully accomplished all of the major program goals, as outlined below.

1) Constructed and tested a communication link that exploited both spatial diversity and temporal interleaving.

2) Demonstrated data transmission using standard network protocols, specifically, SONET OC-48 encapsulated in OTU1.

3) Demonstrated error-free data transmission at the design range of $30 \mathrm{~km}$ and maintained link functionality out to $60 \mathrm{~km}$.

4) Accomplished error-free transfers of 6-minute, 100 Gbyte image files during several test flights.

5) Quantified link performance and channel conditions as a function of time-of-day, altitude, elevation angle, and propagation range.

These data will serve as a basis for future free-space link constructs. A primary goal of the next link demonstration is the incorporation of frame retransmission, which will be designed to be error tolerant in situations where the frame loss is as high as $10 \%$.

\section{REFERENCES}

[1] J. W. Hardy, et al., "Real-Time Atmospheric Compensation," J. Opt. Soc. Am., vol. 67, p. 360 (1977).

[2] S. Trisno, et al., "Characterization of Delayed Diversity Optical Wireless System to Mitigate Atmospheric Turbulence Induced Fading," Proc. SPIE, vol. 5892 (2005).

[3] I. S. Reed and G. Solomon, "Polynomial Codes Over Certain Finite Fields," J. Soc. Ind. Appl. Math., vol. 8, p. 300 (1960).

[4] B. Vucetic and J. Yuan, Turbo Codes: Principles and Applications, Kluwer Academic Publishers (2000).

[5] M. Northcott, et al., "Long Distance Laser Communications Demonstration," Proc. SPIE, vol. 6578 (2007).
[6] L. B. Stotts, et al., "Hybrid Optical RF Airborne Communications," Proc. IEEE, vol. 97. p. 1109 (2009).

[7] J. D. Moores, et al., "Architecture Overview and Data Summary of a $5.4 \mathrm{~km}$ Free-Space Laser Communications Experiment," Proc. SPIE, vol. 7464 (2009).

[8] J. G. Proakis and M. Salehi, Digital Communications, McGraw-Hill (2008).

[9] J. A. Greco, "Design of the High-Speed Framing, FEC, and Interleaving Hardware Used in a $5.4 \mathrm{~km}$ Free-Space Optical Communication Experiment," Proc.SPIE, vol. 7464 (2009).

[10] R. J. Murphy, et al., "A Conical Scan Free Space Optical Tracking System for Fading Channels," Proc. SPIE, vol. 7464 (2009). 